\title{
Duloxetine reduces pain after Total hip arthroplasty: a prospective, randomized controlled study
}

\author{
Hao Li ${ }^{1+}$, Wei-Nan Zeng ${ }^{1,2+}$, Zi-Chuan Ding ${ }^{1}$, Ming-Cheng Yuan ${ }^{1}$, Yong-Rui Cai ${ }^{1}$ and Zong-Ke Zhou ${ }^{1 *}$
}

\begin{abstract}
Background: Previous studies have demonstrated the efficacy of duloxetine in reducing postoperative pain and opioid consumption. However, the effect of duloxetine on total hip arthroplasty (THA) remains unclear. The objective of this study was to assess the efficacy of oral duloxetine in THA.

Methods: We enrolled 96 patients in this randomized controlled trial. These patients were randomized $(1,1)$ to either the duloxetine group or the placebo group and received daily doses of $60 \mathrm{mg}$ duloxetine or placebo, respectively, from $2 \mathrm{~d}$ pre-operation to $14 \mathrm{~d}$ after surgery. The primary outcome was pain severity upon movement measured by a visual analogue scale (VAS). The secondary outcomes included VAS scores for resting pain, morphine consumption, Harris Hip Score, patient satisfaction at discharge, length of postoperative hospital stay, and adverse events.

Results: Patients in the duloxetine group had significantly lower pain severity scores upon movement within 3 postoperative weeks $(p<0.05)$ while none of the differences met the minimum clinically important difference (MCID). Moreover, patients in the duloxetine group performed better in terms of resting pain (in 3 weeks after surgery), morphine requirements, and satisfaction level at discharge (all $p<0.05)$. There was no difference between groups in the prevalence of adverse events.

Conclusions: Although it did not result in a clinically meaning reduction in pain after total hip arthroplasty, perioperative administration of $60 \mathrm{mg}$ of duloxetine daily significantly alleviated pain in the postoperative 3 weeks and morphine requirements during the postoperative $48 \mathrm{~h}$. Therefore, duloxetine still shows promise in optimizing the multimodal pain-management protocols in total hip arthroplasty.
\end{abstract}

Trial registration: Chinese Clinical Trial Registry, ChiCTR2000033606, 06/06/2020.

Keywords: Duloxetine, Total hip arthroplasty, Postoperative pain

\footnotetext{
* Correspondence: zhouzongke@scu.edu.cn

${ }^{\dagger} \mathrm{Hao}$ Li and Wei-Nan Zeng contributed equally to this work.

'Department of Orthopaedics, West China Hospital, Sichuan University, 37\# WuhouGuoxue Road, Chengdu, China

Full list of author information is available at the end of the article
}

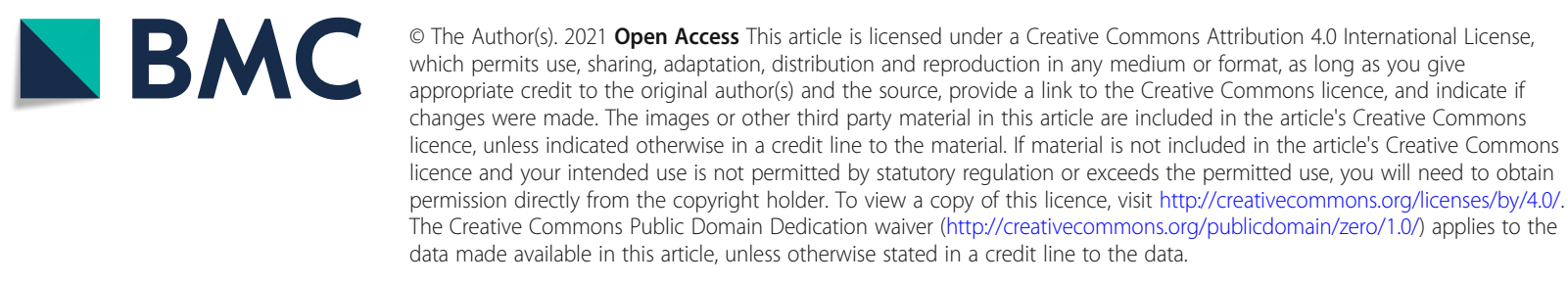




\section{Background}

Total hip arthroplasty (THA) is a common surgical procedure used to successfully, economically, and safely treat end-stage joint diseases that may cause deformities, lower the quality of life, and lead to bodily dysfunction [1]. Despite excellent outcomes, the overall incidence of dissatisfaction associated with THA is relatively high, at approximately $20 \%$ [2].

Approximately 7 to $23 \%$ of THA patients suffer postoperative pain, which is one of the most significant unfavorable outcomes related to this procedure $[3,4]$. Postoperative pain seriously affects perioperative mood, interferes with joint function recovery, prolongs hospitalization, increases medical expenses, and further reduces the quality of life and work [5]. Inadequate perioperative pain management is associated with a distinct possibility of suffering postoperative pain and numerous other serious complications such as joint stiffness, deep vein thrombosis, and pulmonary embolism [5-8].

Severe postoperative pain exacerbates opioid usage and subsequent opioid-related deaths [6]. In 2017, death rates related to overdosing on opioids, including methadone and heroin, rose sharply to 19.3 per 100,000 in the United States [9]. Opioid prescriptions dispensed by orthopedic doctors accounted for a considerable percentage $(7.7 \%)$ of prescriptions [10]. Various multimodal analgesia regimens have been proposed to alleviate postoperative pain and reduce side effects during the use of postoperative opiates. Further optimization of perioperative pain management has always been a major topic of related research efforts $[6,7]$.

Duloxetine (Cymbalta) is a selective serotonin and norepinephrine reuptake inhibitor (SNRI), approved by the Food and Drug Administration (FDA) for the treatment of depression, generalized anxiety disorder, and chronic musculoskeletal pain (osteoarthritis, fibromuscular pain, and chronic back pain, among others) [11, 12]. Duloxetine, which promotes the downregulation of inhibitory pain pathways in the central nervous system, effectively ameliorates pain associated with hyperexcitability corresponding to peripheral sensitization, as well as central sensitization (CS), caused by chronic joint pain $[12,13]$.

However, as far as we know, all previous studies investigating the effects of duloxetine on pain following arthroplasty are focused on total knee arthroplasty (TKA) [12, 14, 15]. To the best of our knowledge, none of the studies have investigated whether duloxetine alleviates postoperative pain following THA. Based on the hypothesis that duloxetine significantly relieves postoperative pain following THA and leads to further positive postoperative outcomes, we conducted a randomized, double-blinded, placebo-controlled study to determine whether duloxetine optimizes perioperative analgesia protocols in THA.

\section{Methods}

A prospective study, in the form of a single-center (West China Hospital of Sichuan University), randomized, double-blinded, parallel-arm, placebo-controlled clinical trial, was conducted. In this study, we enrolled 153 patients who were scheduled for THA from June 2020 to September 2020. The study was approved by the Ethics Committee on Biomedical Research, West China Hospital of Sichuan University (approval no. 2020-843) and registered at the Chinese Clinical Trial Registry (ChiCTR2000033606, 06/06/2020). Informed consent and research authorizations were obtained from all participants. The research report met unified clinical trial reporting standards and conformed with the Declaration of Helsinki [16].

Eligible patients who were over 18 years of age; classified under American Society of Anesthesiologists (ASA) status I, II, or III; and scheduled to undergo primary THA for end-stage hip joint diseases, were screened using the Hamilton Depression Scale (HAMD) and the Hamilton Anxiety Scale (HAMA). Subjects whose HAMD and HAMA scores were both $<7$ were included. The exclusion criteria were as follows: a known allergy to any of the studied drugs, previous exposure to SNRIs or selective serotonin reuptake inhibitors; known psychiatric disorders; alcohol or opioid abuse; acute infections of the hip joint; recent treatment for malignant diseases; major previous ipsilateral hip arthroplasty or open surgery; peripheral or central nerve impairment; cognitive dysfunction; history of peptic ulcers or bleeding tendency; impaired liver and/or renal function; and poor physical condition indicating the lack of ability to tolerate surgery.

Sample size estimation was determined based on previous studies [12,17]. A sample size of 48 patients in each group was required to test this 2-tailed hypothesis at a power of 0.80, an alpha level of 0.05 , and a dropout rate of $20 \%$ for detecting a 2 -point difference in the pain severity score between groups following surgery. The pain severity score was evaluated using a visual analog scale (VAS) consisting of a horizontal line divided into 10 equal parts. The ends of the horizontal line were marked " 0 " and " 10 " and were used to represent no pain to severe pain, respectively; the middle area represented different degrees of pain. The 2-point difference was determined to be the minimum clinically important difference (MCID) because the average acceptable VAS pain score difference following surgery was approximately 2 points according previous studies $[12,18,19]$.

Participants were randomized $(1,1)$ to either the duloxetine group or the placebo group. Randomization was 
concealed from researchers as well as from the patients, by way of sealed envelopes delivered following hospitalization. A sealed, opaque envelope, containing the randomized grouping plan was prepared in advance. After an eligible patient was assigned a sickbed and excluded from surgical contraindications, an independent researcher opened the randomized envelope in the order in which the patients were enrolled to determine the grouping of that patient. Neither the participants nor the primary investigator was aware of the grouping status until the data analysis stage at the end of the study. The hospital pharmacy prepared two types of indistinguishable capsules containing either $60 \mathrm{mg}$ of duloxetine or a placebo (starch) for daily oral administration starting from $2 \mathrm{~d}$ pre-surgeryto $14 \mathrm{~d}$ post-surgery. Surgery was performed by the same senior doctor, an experienced surgeon who had performed over 300 THA annually. All participants accepted a multimodal and standardized analgesic strategy. From preoperative day 2 to the day before surgery, every patient was given celecoxib $200 \mathrm{mg}$ twice a day (one dose after breakfast and one dose after dinner) for preemptive analgesia. During the operation, all patients received general anesthesia, which was composed of an induction of sufentanil $0.5 \mu \mathrm{g} / \mathrm{kg}$, midazolam $0.04 \mathrm{mg} / \mathrm{kg}$, propofol $1-2 \mathrm{mg} / \mathrm{kg}$ and cistracurium $2 \mu \mathrm{g} /$ $\mathrm{kg}$ intravenously, and a following continuous intravenous infusion of $0.1-0.3 \mu \mathrm{g} /(\mathrm{kg} \cdot \mathrm{min})$ of remifentanil, $2-5$ $\mathrm{mg} /(\mathrm{kg} \bullet \mathrm{h})$ of propofol and inhalation of sevoflurane to maintain anesthesia. Besides, all patients were treated with an $80 \mathrm{~mL}$ periarticular injection of $0.25 \%$ ropivacaine for local infiltration analgesia. Since postoperative day 1 , every patient restarted oral administration of celecoxib (200 mg twice a day) until 2 weeks after the surgery. When acute pain was unbearable or VAS was $>6$, morphine (5 mg intravenously) was used as a rescue analgesic $[20,21]$. In the perioperative period, there was no other oral analgesics except celecoxib, and intravenous morphine was the only rescue analgesic before discharge. Patients' discharge criteria for postoperative pain included: pain must be tolerable without affecting daily life and rehabilitation, and the severity of acute pain no longer required intravenous morphine to relieve. All participants were followed up for 3 months.

The pain severity score upon movement $(3 \mathrm{~h}, 6 \mathrm{~h}, 12 \mathrm{~h}$, $24 \mathrm{~h}, 48 \mathrm{~h}, 72 \mathrm{~h}, 1 \mathrm{w}, 3 \mathrm{w}$, and $3 \mathrm{~m}$ following surgery) was considered as the primary outcome, because movementevoked pain during the postoperative period is more severe, more frequent, and exerts a greater influence on postoperative functional rehabilitation compared with pain at rest [22]. Secondary outcomes included pain severity scores at rest $(3 \mathrm{~h}, 6 \mathrm{~h}, 12 \mathrm{~h}, 24 \mathrm{~h}, 48 \mathrm{~h}, 72 \mathrm{~h}, 1 \mathrm{w}$, $3 \mathrm{w}$, and $3 \mathrm{~m}$ following surgery), morphine consumption (24 h, $72 \mathrm{~h}$, and $1 \mathrm{w}$ following surgery), Harris Hip Score (HHS; $3 \mathrm{w}$ and $3 \mathrm{~m}$ following surgery), patient satisfaction at discharge, length of postoperative hospital stay and adverse events. Participants were requested to complete a 7-point satisfaction questionnaire before discharge [23]. Satisfaction levels ranged from being extremely satisfied to extremely dissatisfied. Adverse events were recorded until the last day on which duloxetine or the placebo was administered.

All data management and statistical analyses were conducted using IBM, SPSS version 22.0 software. Whereas the independent $\mathrm{t}$-test was used to analyze differences between continuous variables, such as body mass index (BMI) and age, the chi-square test or Fisher's exact test was used to analyze categorical variables. The significance level was set at $p<0.05$.

\section{Results}

A total of 153 patients with end-stage joint diseases were scheduled to undergo a primary unilateral THA procedure during the recruitment period (June 2020 to September 2020) at our department. Among these 153 patients, 49 did not satisfy the inclusion criteria and 8 declined to participate in the study. Thus, 96 eligible patients were included in the study. These patients were randomly assigned to either the duloxetine group or the placebo group. Specific information regarding patient flow is plotted (Fig. 1). There were no significant differences in the preoperative demographics and characteristics of the study participants between these groups (Table 1; $p>$ 0.05).

The pain severity scores upon movement within the 3 postoperative weeks in patients in the duloxetine group were significantly lower compared to those in the placebo group (Table 2; $p<0.05$ ). However, none of the between-group differences exceeds the MCID, and there was no significant difference between the pain severity scores upon movement in patients of the duloxetine and placebo groups after 3 months of surgery.

Similar results were obtained for resting pain, wherein the post-operative movement-evoked pain level in patients of the duloxetine group was significantly lower than those in the placebo group until postoperative week 3 (Table $3 ; p<0.05$ ). There were no significant differences between the VAS scores for resting pain of the 2 groups after 3 months of surgery. Postoperative morphine consumption and HSS until $24 \mathrm{~h}, 72 \mathrm{~h}$, and $1 \mathrm{w}$ after surgery are shown in Table 4. Patients in the duloxetine group required significantly less morphine compared to those in the placebo group $(p<0.05)$. There was no significant difference in HSS between the duloxetine group and the placebo group at either $21 \mathrm{~d}$ or 3 months after surgery. In terms of the satisfaction level at discharge, 45 patients in the duloxetine group expressed satisfaction (defined as extremely, very, or somewhat satisfied) with the treatment, compared to 36 patients in 


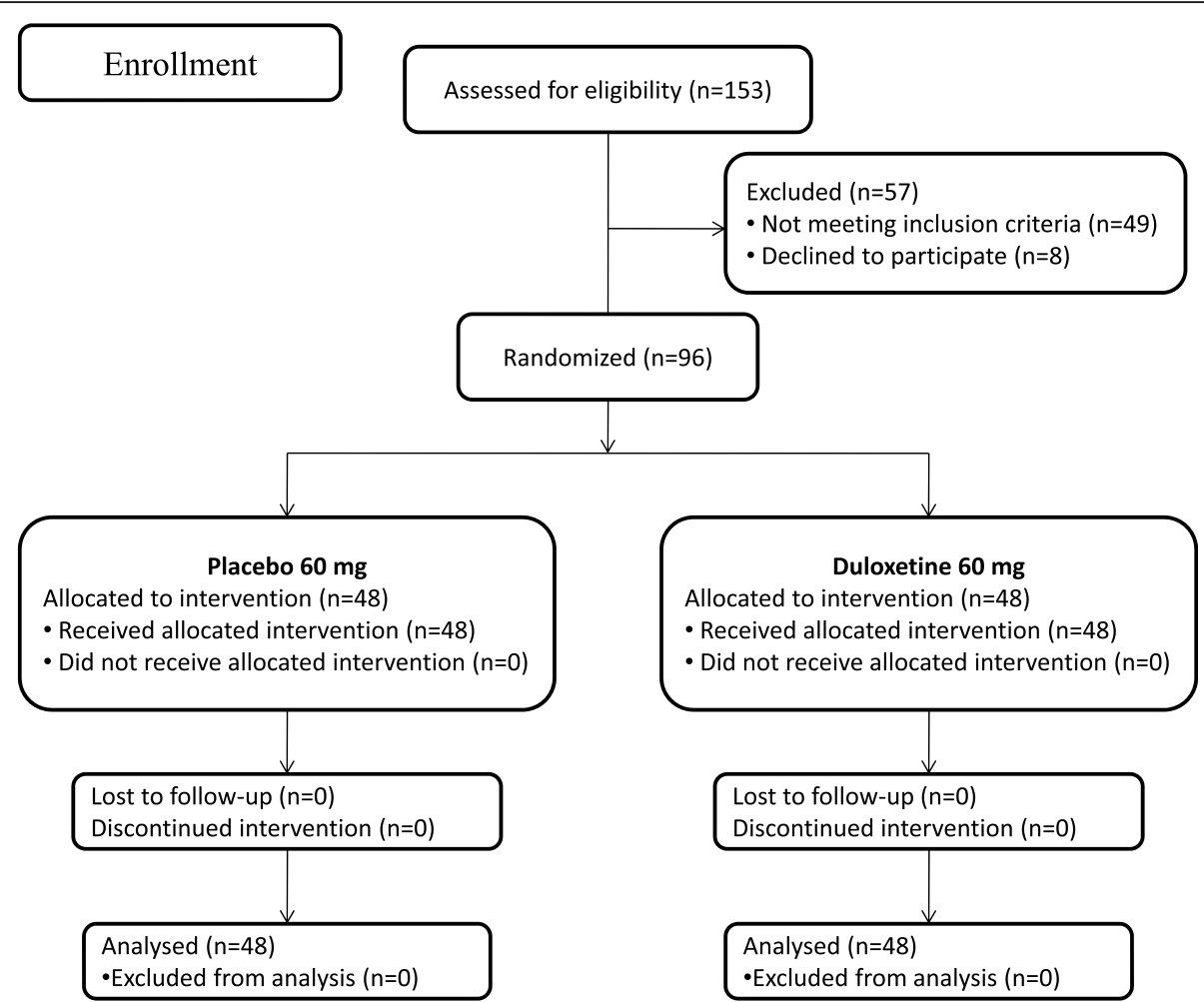

Fig. 1 Schematic of the study design

the placebo group ( $94 \%$ versus $75 \%, p=0.011$ ). No significant difference was observed between the lengths of postoperative hospital stay between the two groups. There was no significant difference in the adverse event between groups (Table $5 ; p>0.05$ ).

\section{Discussion}

In this trial, perioperative daily oral administration of 60 mg duloxetine from $2 \mathrm{~d}$

pre-operation to $14 \mathrm{~d}$ after surgery resulted in the lowering of postoperative movement-evoked pain and resting pain as well as in morphine requirements, while the pain differences between two groups are below the MCID. Moreover, patients expressed an increased satisfaction level at discharge from the hospital. Duloxetine did not appear to increase the incidence of adverse events.

The use of oral duloxetine in arthroplasty has been studied previously. Some of these studies have demonstrated its efficacy in reducing postoperative pain and opioid consumption [12, 14, 15]. As far as we know, all studies that have been conducted to determine the efficacy of duloxetine in alleviating residual pain following total joint replacement have focused on TKA and not on THA. Thus, the effect of orally administered duloxetine on THA remains unclear. Therefore, in an attempt to resolve the aforementioned issue, we conducted a randomized, controlled study to validate the effect of duloxetine on THA.

Pain, which follows arthroplasty, is a serious complication that always confounds orthopedists. Most recent literature indicates that long-lasting, intense, harmful pain stimuli induced by chronic joint diseases may trigger peripheral nociceptors and upregulate the excitability as well as synaptic efficacy of neurons in the central nociceptive pathways, leading to central sensitization. Central sensitization manifests as pain hypersensitivity and further triggers severe postoperative pain following arthroplasty [12, 13, 24-26]. Because serotonin signaling is involved in pain processing [27], the efficacy of SNRIs, including duloxetine, in resolving postoperative pain has been investigated [15].

In this study, we found that perioperative administration of duloxetine effectively relieved movement-evoked pain and resting pain 3 weeks post-surgery. These results are partially substantiated by a previously conducted randomized controlled trial, in which 80 patients scheduled for TKA, who were treated with duloxetine, achieved a better analgesic effect during the postoperative period from 2 to 12 weeks [12]. Except for the intrinsic difference between THA and TKA, the reason for the difference between the postoperative periods required to relieve pain may be partly attributed to the differences in the duration of the oral administration of 
Table 1 Preoperative demographics and characteristics

\begin{tabular}{|c|c|c|c|}
\hline & Duloxetine $(n=48)$ & Placebo $(n=48)$ & $P$ value \\
\hline \multicolumn{4}{|l|}{ Demographic data } \\
\hline $\operatorname{Age}^{a}(y r)$ & $52.7 \pm 12.0$ & $50.2+13.2$ & 0.333 \\
\hline Male sex ${ }^{\mathrm{b}}$ (no.[\%] of patients) & $22(46 \%)$ & $24(50 \%)$ & 0.683 \\
\hline$B M l^{a}\left(k g / m^{2}\right)$ & $24.0 \pm 2.9$ & $23.9 \pm 3.4$ & 0.890 \\
\hline \multicolumn{4}{|l|}{ ASA status ${ }^{\mathrm{b}}$ (no.[\%] of patients) } \\
\hline । & $8(17 \%)$ & $4(8 \%)$ & \\
\hline$\|$ & $28(58 \%)$ & $32(67 \%)$ & \\
\hline III & $12(25 \%)$ & $12(25 \%)$ & \\
\hline Surgical site (right/left) & $27 / 21$ & $24 / 24$ & \\
\hline \multicolumn{4}{|l|}{ Diagnosis ${ }^{\mathrm{b}}$ (no.of patients) } \\
\hline Osteonecrosis of femoral head & $16(33 \%)$ & $17(35 \%)$ & \\
\hline Primary osteoarthritis & 15 (31\%) & $13(27 \%)$ & \\
\hline Developmental dysplasia of the hip & $9(19 \%)$ & $10(21 \%)$ & \\
\hline Others & $8(17 \%)$ & $8(17 \%)$ & \\
\hline \multicolumn{4}{|l|}{ Preoperative parameters $^{a}$} \\
\hline \multicolumn{4}{|l|}{ hip function } \\
\hline Flexion $\left(^{\circ}\right)$ & $91.7 \pm 11.8$ & $90.2 \pm 15.8$ & 0.610 \\
\hline Abduction $\left(^{\circ}\right)$ & $21.5 \pm 7.3$ & $21.6 \pm 8.4$ & 0.948 \\
\hline Harris Hip Score (points) & $42.1 \pm 9.2$ & $41.2 \pm 10.6$ & 0.652 \\
\hline \multicolumn{4}{|l|}{ BPI-pain severity score } \\
\hline Average & $5.4 \pm 1.3$ & $5.3 \pm 1.1$ & 0.666 \\
\hline Worst & $7.6 \pm 1.4$ & $7.2 \pm 1.7$ & 0.128 \\
\hline Least & $2.7 \pm 1.2$ & $2.7 \pm 1.1$ & 0.861 \\
\hline Current & $5.4 \pm 1.6$ & $5.1 \pm 1.2$ & 0.243 \\
\hline \multicolumn{4}{|l|}{ BPI-interference score } \\
\hline General activity & $6.5 \pm 2.0$ & $6.5 \pm 2.3$ & 0.925 \\
\hline Walking & $7.3 \pm 1.9$ & $7.4 \pm 2.5$ & 0.853 \\
\hline Work & $6.5 \pm 2.1$ & $7.3 \pm 2.3$ & 0.082 \\
\hline Sleep & $5.0 \pm 2.1$ & $4.5 \pm 2.3$ & 0.264 \\
\hline Relations with others & $5.0 \pm 2.3$ & $4.7 \pm 1.6$ & 0.408 \\
\hline Enjoyment of life & $4.8 \pm 1.5$ & $4.5 \pm 1.6$ & 0.430 \\
\hline Mood & $5.2 \pm 2.0$ & $5.5 \pm 2.4$ & 0.512 \\
\hline HAMD & $3.3 \pm 1.3$ & $3.0 \pm 1.4$ & 0.246 \\
\hline HAMA & $3.4 \pm 1.4$ & $3.2 \pm 1.4$ & 0.425 \\
\hline
\end{tabular}

BPI Brief Pain Inventory

${ }^{a}$ Data are given as the mean \pm standard deviation

${ }^{b}$ Data are given as the number (percentage) of patients

duloxetine as well as its dosage. In our study, patients in the duloxetine group were administered duloxetine starting $2 \mathrm{~d}$ pre-surgery to $14 \mathrm{~d}$ post-surgery, as opposed to a previous study, which followed a protocol of administering $30 \mathrm{mg}$ of duloxetine orally on the night before surgery and $30 \mathrm{mg}$ per day for 6 weeks post-surgery. The Cochrane database review and other published literature have indicated that $60 \mathrm{mg}$ of duloxetine administered daily was effective in treating painful neuropathy or chronic pain, whereas daily doses lower than $60 \mathrm{mg}$ were ineffective [14, 15, 28]. Clinical pharmacokinetic studies reveal that duloxetine achieves maximum plasma concentration approximately $6 \mathrm{~h}$ after dosing and that its biological half-life is approximately $10-12 \mathrm{~h}$ [29]. Therefore, the protocol used for the perioperative administering of duloxetine in our study is believed to be 
Table 2 Primary outcomes regarding VAS scores upon the movement

\begin{tabular}{llll}
\hline $\begin{array}{l}\text { Time after } \\
\text { surgery }\end{array}$ & $\begin{array}{l}\text { Duloxetine* } \\
(\boldsymbol{n}=\mathbf{4 8 )}\end{array}$ & $\begin{array}{l}\text { Placebo* } \\
(\boldsymbol{n}=\mathbf{4 8})\end{array}$ & $\boldsymbol{P}$ valuet \\
\hline $3 \mathrm{~h}$ & $6.0 \pm 0.9$ & $6.5 \pm 1.2$ & $\mathbf{0 . 0 2 9}$ \\
$6 \mathrm{~h}$ & $5.9 \pm 0.8$ & $6.4 \pm 1.0$ & $\mathbf{0 . 0 1 6}$ \\
$12 \mathrm{~h}$ & $5.4 \pm 0.9$ & $5.9 \pm 1.0$ & $\mathbf{0 . 0 1 0}$ \\
$24 \mathrm{~h}$ & $5.0 \pm 1.0$ & $5.5 \pm 1.2$ & $\mathbf{0 . 0 3 3}$ \\
$48 \mathrm{~h}$ & $4.4 \pm 1.0$ & $4.8 \pm 1.2$ & $\mathbf{0 . 0 4 1}$ \\
$72 \mathrm{~h}$ & $3.9 \pm 0.9$ & $4.5 \pm 1.1$ & $\mathbf{0 . 0 0 4}$ \\
$1 \mathrm{~W}$ & $2.8 \pm 1.0$ & $3.5 \pm 1.1$ & $<\mathbf{0 . 0 0 1}$ \\
$3 \mathrm{~W}$ & $1.9 \pm 1.0$ & $2.5 \pm 1.1$ & $\mathbf{0 . 0 0 7}$ \\
$3 \mathrm{~m}$ & $1.6 \pm 1.0$ & $1.8 \pm 1.0$ & 0.469 \\
\hline
\end{tabular}

*The values are given as the mean and standard deviation. $+P$ values are calculated by independent t-test. $P$ values indicating a significant difference among groups are in bold

appropriate and adequate. MCID is an important concept to put in perspective statistically significant results that may not be clinically relevant [30]. According to previous studies, we used an MCID for the VAS score of 2 in our study, while there were also other studies that determined MCID as other values [12, 18, 19]. To reduce the occurrence of side effects or complications, an analgesia protocol should preferably be multimodal [31]. When using a multimodal analgesia protocol, it is raising the bar very high to identify the performance of individual intervention, therefore, the findings which did not reach the MCID should have a role in a multimodal pain control protocol [31-33]. In a prospective, randomized controlled study comparing local infiltration anesthetic and control, authors found that the local analgesia group had a significantly lower mean VAS score for pain during exercise than did the control group (4.7 vs 6.6) on

Table 3 Secondary outcomes regarding VAS scores for resting pain

\begin{tabular}{llll}
\hline $\begin{array}{l}\text { Time after } \\
\text { surgery }\end{array}$ & $\begin{array}{l}\text { Duloxetine* } \\
(\boldsymbol{n}=\mathbf{4 8})\end{array}$ & $\begin{array}{l}\text { Placebo* } \\
(\boldsymbol{n}=\mathbf{4 8})\end{array}$ & $\boldsymbol{P}$ valuet \\
\hline $3 \mathrm{~h}$ & $4.1 \pm 1.0$ & $4.5 \pm 1.1$ & $\mathbf{0 . 0 2 6}$ \\
$6 \mathrm{~h}$ & $4.0 \pm 1.0$ & $4.4 \pm 1.0$ & $\mathbf{0 . 0 3 2}$ \\
$12 \mathrm{~h}$ & $3.3 \pm 1.0$ & $3.8 \pm 1.1$ & $\mathbf{0 . 0 4 4}$ \\
$24 \mathrm{~h}$ & $3.0 \pm 1.1$ & $3.5 \pm 1.1$ & $\mathbf{0 . 0 4 6}$ \\
$48 \mathrm{~h}$ & $2.3 \pm 1.0$ & $2.8 \pm 1.0$ & $\mathbf{0 . 0 1 9}$ \\
$72 \mathrm{~h}$ & $1.8 \pm 0.9$ & $2.2 \pm 1.0$ & $\mathbf{0 . 0 2 3}$ \\
$1 \mathrm{~W}$ & $1.0 \pm 0.8$ & $1.3 \pm 0.8$ & $\mathbf{0 . 0 4 0}$ \\
$3 \mathrm{~W}$ & $0.8 \pm 0.7$ & $1.1 \pm 0.7$ & $\mathbf{0 . 0 4 3}$ \\
$3 \mathrm{~m}$ & $0.6 \pm 0.5$ & $0.7 \pm 0.6$ & 0.851 \\
\hline
\end{tabular}

*The values are given as the mean and standard deviation. $+P$ values are calculated by independent t-test. $P$ values indicating a significant difference among groups are in bold
Table 4 Secondary outcomes regarding morphine consumption and HHS

\begin{tabular}{cccc}
\hline & Duloxetine* $(\boldsymbol{n}=\mathbf{4 8})$ & Placebo* $^{*}(\boldsymbol{n}=\mathbf{4 8})$ & $\boldsymbol{P}$ valuef \\
\hline Morphine Consumption & & \\
PO 24h & $11.0 \pm 4.9$ & $14.2 \pm 5.9$ & $\mathbf{0 . 0 0 6}$ \\
PO 72 h & $16.8 \pm 6.1$ & $20.4 \pm 9.8$ & $\mathbf{0 . 0 3 2}$ \\
PO 1w & $18.7 \pm 7.3$ & $23.3 \pm 13.6$ & $\mathbf{0 . 0 3 9}$ \\
HHS & & & \\
PO 3w & $75.4 \pm 5.5$ & $73.7 \pm 6.1$ & 0.156 \\
PO 3 m & $87.2 \pm 4.7$ & $87.8 \pm 4.4$ & 0.517
\end{tabular}

$P O$ postoperative. ${ }^{*}$ The values are given as the mean and standard deviation. $+P$ values are calculated by independent t-test. $P$ values indicating a significant difference among groups are in bold

the first day after surgery $(p=0.008)$, and the difference for the VAS score of 1.9 offered improved pain control [31].

The findings of our study indicated that duloxetine reduced morphine consumption within $1 \mathrm{w}$ postTHA. Two previous randomized controlled trials that enrolled 106 and 50 participants, respectively, also showed similar results $[14,15]$. In one of the former studies, a daily oral dose of $60 \mathrm{mg}$ duloxetine was administered to patients approximately $30 \mathrm{~min}$ prior to them being transferred to the operating room. The treatment was continued until 2 weeks after surgery and was found to significantly reduce the total opioid requirements for over a period of 3 months [15]. The other study revealed that two oral doses of $60 \mathrm{mg}$ duloxetine, administered $2 \mathrm{~h}$ before surgery and on the first day after surgery, reduced morphine consumption in the first $48 \mathrm{~h}$ post-TKA [14]. The inconsistencies observed during the postoperative periods that warranted the reduction of opioid requirements in these two studies may be owing to the differences in assessment times as well as the dosage of duloxetine administered, which in turn are associated with the half-life of duloxetine.

The decision to use $60 \mathrm{mg}$ of duloxetine as the daily dose in our study was based on the reports of previous studies [14, 15, 28]. Adequate quality-based evidence indicates that a daily dose of $60 \mathrm{mg}$ of duloxetine is indeed the appropriate dosage [28]. Lower daily doses of duloxetine are not efficacious in alleviating pain; moreover, higher daily doses do not improve its efficacy and may even result in adverse events [28]. Our results also demonstrated that duloxetine did not increase the incidence of adverse events at a daily dose of $60 \mathrm{mg}$.

Our study had a few limitations. First, the 3-month follow-up time may obscure the long-term safety of duloxetine. However, the biological half-life of 
Table 5 Secondary outcomes regarding satisfaction level, length of PO hospital stays and adverse events

\begin{tabular}{|c|c|c|c|}
\hline & Duloxetine $(n=48)$ & Placebo $(n=48)$ & $P$ value \\
\hline \multicolumn{4}{|l|}{ Satisfaction levelt (no.[\%] of patients) } \\
\hline Extremely satisfied & $19(40 \%)$ & $11(23 \%)$ & \\
\hline Very satisfied & $21(44 \%)$ & 17 (35\%) & \\
\hline Somewhat satisfied & $5(10 \%)$ & $8(17 \%)$ & \\
\hline Neither satisfied nor dissatisfied & $2(4 \%)$ & $6(13 \%)$ & \\
\hline Somewhat dissatisfied & $1(2 \%)$ & $4(8 \%)$ & \\
\hline Very dissatisfied & 0 & $2(4 \%)$ & \\
\hline Extremely dissatisfied & 0 & 0 & \\
\hline length of PO hospital stays ${ }^{\mathrm{a}}(\mathrm{h})$ & $63.4 \pm 17.6$ & $70.7 \pm 23.7$ & 0.089 \\
\hline \multicolumn{4}{|l|}{ Adverse Eventst (no.[\%] of patients) } \\
\hline Nausea and vomiting & $8(17 \%)$ & $7(15 \%)$ & 0.779 \\
\hline Dry mouth & $4(8 \%)$ & $2(4 \%)$ & 0.677 \\
\hline Insomnia & $5(10 \%)$ & $6(13 \%)$ & 0.749 \\
\hline Somnolence & $6(13 \%)$ & $3(6 \%)$ & 0.486 \\
\hline Constipation & $7(15 \%)$ & $9(19 \%)$ & 0.584 \\
\hline Dizziness & $3(6 \%)$ & $4(8 \%)$ & 1.000 \\
\hline Fatigue & $5(10 \%)$ & $7(15 \%)$ & 0.537 \\
\hline
\end{tabular}

${ }^{\mathrm{a}}$ Data are given as the mean \pm standard deviation. + Data are given as the number (percentage) of patients

duloxetine is approximately $10-12 \mathrm{~h}$, and maximum plasma concentration is attained approximately $6 \mathrm{~h}$ after dosing [29]. Patients with impaired liver and renal function were excluded during screening; therefore, the 3-month follow-up period was adequate for observing and treating adverse events. A second limitation was that the trial was performed at a single center, which may reduce generalizability. Nevertheless, generalizability is also influenced by different factors such as centers and individuals, as well as by inclusion and exclusion criteria. Lastly, the initial sample size estimation was based on our primary outcome, implying that this sample size may not be appropriate for determining a significant difference in terms of all relevant outcomes.

\section{Conclusions}

In conclusion, the perioperative daily administration of $60 \mathrm{mg}$ duloxetine to patients undergoing primary unilateral total hip arthroplasty alleviated movement-evoked pain 3 weeks post-surgery, although it did not result in a clinically meaning reduction in postoperative pain. Duloxetine also alleviated resting pain within 3 postoperative weeks, reduced morphine requirements following surgery, and improved satisfaction levels at discharge, without increasing the incidence of adverse events. Considering the positive results of the current study, it may be concluded that duloxetine shows potential as a novel therapeutic agent and could likely be used to optimize multimodal pain management protocols in total hip arthroplasty.

\section{Abbreviation \\ THA: Total hip arthroplasty; SNRI: Selective serotonin and norepinephrine reuptake inhibitor; FDA: Food and Drug Administration; CS: Central sensitization; TKA: Total knee arthroplasty; ASA: American Society of Anesthesiologists; HAMD: Hamilton Depression Scale; HAMA: Hamilton Anxiety Scale; VAS: Visual analog scale; MCID: Minimum clinically important difference; HHS: Harris Hip Score; BMI: Body mass index}

\section{Acknowledgements \\ Not applicable.}

\section{Authors' contributions}

HL: study design and writing; WNZ: data collections and writing; ZCD: data collections and data analysis; MCY: data collections and data analysis; YRC: writing; ZKZ: study design. All authors read and approved the final manuscript.

\section{Funding}

This work was supported by 1.3.5 project for disciplines of excellence, West China Hospital, Sichuan University (ZYJC18039) and Regional Innovation \& Cooperation program of Science \& Technology Department of Sichuan Province (No. 2021YFQ0028)

\section{Availability of data and materials}

The datasets used and/or analysed during the current study are available from the corresponding author on reasonable request.

\section{Declarations}

\section{Ethics approval and consent to participate}

The study was approved by the Ethics Committee on Biomedical Research, West China Hospital of Sichuan University (approval no.2020-843). All procedures performed in studies involving human participants were in 
accordance with the ethical standards of the institutional and/or national research committee and with the 1964 Helsinki declaration and its later amendments or comparable ethical standards. This study was approved by the institutional review board. Informed consent was obtained from all individual participants included in the study. Additional informed consent was obtained from all individual participants for whom identifying information is included in this article.

\section{Consent for publication}

Written informed consent for publication was obtained from all participants.

\section{Competing interests}

The authors declare that they have no competing interests.

\section{Author details}

'Department of Orthopaedics, West China Hospital, Sichuan University, 37\# WuhouGuoxue Road, Chengdu, China. ${ }^{2}$ Department of Orthopaedics, Chongqing General Hospital, University of Chinese Academy of Sciences, Chongqing, China.

Received: 18 February 2021 Accepted: 18 May 2021

Published online: 28 May 2021

\section{References}

1. Mercurio M, Gasparini G, Carbone EA, Galasso O, Segura-Garcia C. Personality traits predict residual pain after total hip and knee arthroplasty. Int Orthop. 2020;44(7):1263-70.

2. Goodman SM, Mehta BY, Kahlenberg CA, Krell EC, Nguyen J, Finik J, et al. Assessment of a satisfaction measure for use after primary total joint arthroplasty. J Arthroplast. 2020;35(7):1792-9 e1794.

3. Beswick AD, Wylde V, Gooberman-Hill R, Blom A, Dieppe P. What proportion of patients report long-term pain after total hip or knee replacement for osteoarthritis? A systematic review of prospective studies in unselected patients. BMJ Open. 2012;2(1):e000435

4. Anakwe RE, Jenkins PJ, Moran M. Predicting dissatisfaction after total hip arthroplasty: a study of 850 patients. J Arthroplast. 2011;26(2):209-13.

5. Parvizi J, Miller AG, Gandhi K. Multimodal pain management after total joint arthroplasty. J Bone Joint Surg Am. 2011;93(11):1075-84.

6. Padilla JA, Gabor JA, Schwarzkopf R, Davidovitch RI. A novel opioid-sparing pain management protocol following total hip arthroplasty: effects on opioid consumption, pain severity, and patient-reported outcomes. J Arthroplast. 2019;34(11):2669-75.

7. Hannon CP, Keating TC, Lange JK, Ricciardi BF, Waddell BS, Della Valle CJ. Anesthesia and analgesia practices in total joint arthroplasty: a survey of the american association of hip and knee surgeons membership. J Arthroplast. 2019:34(12):2872-7 e2872.

8. Joshi GP, Ogunnaike BO. Consequences of inadequate postoperative pain relief and chronic persistent postoperative pain. Anesthesiol Clin North Am. 2005;23(1):21-36.

9. Hedegaard H, Miniño AM, Warner M. Drug overdose deaths in the united states, 1999-2017. NCHS data brief, 2018;329:1-8.

10. Volkow ND, McLellan TA, Cotto JH, Karithanom M, Weiss SR. Characteristics of opioid prescriptions in 2009. Jama. 2011;305(13):1299-301.

11. Chappell AS, Ossanna MJ, Liu-Seifert H, lyengar S, Skljarevski V, Li LC, et al. Duloxetine, a centrally acting analgesic, in the treatment of patients with osteoarthritis knee pain: a 13-week, randomized, placebo-controlled trial. Pain. 2009;146(3):253-60.

12. Koh IJ, Kim MS, Sohn S, Song KY, Choi NY, In Y. Duloxetine reduces pain and improves quality of recovery following total knee arthroplasty in centrally sensitized patients: a prospective, randomized controlled study. J Bone Joint Surg Am. 2019:101(1):64-73.

13. Blikman T, Rienstra W, van Raaij TM, ten Hagen AJ, Dijkstra B, Zijlstra WP, et al. Duloxetine in osteoarthritis (doa) study: study protocol of a pragmatic open-label randomised controlled trial assessing the effect of preoperative pain treatment on postoperative outcome after total hip or knee arthroplasty. BMJ Open. 2016;6(3):e010343.

14. Ho KY, Tay W, Yeo MC, Liu H, Yeo SJ, Chia SL, et al. Duloxetine reduces morphine requirements after knee replacement surgery. Br J Anaesth. 2010; 105(3):371-6.

15. YaDeau JT, Brummett CM, Mayman DJ, Lin Y, Goytizolo EA, Padgett DE, et al. Duloxetine and subacute pain after knee arthroplasty when added to a multimodal analgesic regimen: a randomized, placebo-controlled, tripleblinded trial. Anesthesiology. 2016;125(3):561-72.

16. Schulz KF, Altman DG, Moher D. Consort 2010 statement: updated guidelines for reporting parallel group randomised trials. J Clin Epidemiol. 2010;63(8):834-40

17. Koh HJ, Koh IJ, Kim MS, Choi KY, Jo HU, In Y. Does patient perception differ following adductor canal block and femoral nerve block in total knee arthroplasty? A simultaneous bilateral randomized study. J Arthroplast. 2017; 32(6):1856-61.

18. Myles PS, Urquhart N. The linearity of the visual analogue scale in patients with severe acute pain. Anaesth Intensive Care. 2005:33(1):54-8.

19. Myles PS, Myles DB, Galagher W, Boyd D, Chew C, MacDonald N, et al. Measuring acute postoperative pain using the visual analog scale: the minimal clinically important difference and patient acceptable symptom state. Br J Anaesth. 2017;118(3):424-9.

20. Ding ZC, Xu B, Liang ZM, Wang HY, Luo ZY, Zhou ZK. Limited influence of comorbidities on length of stay after total hip arthroplasty: experience of enhanced recovery after surgery. Orthop Surg. 2020;12(1):153-61.

21. Li D, Wang Q, Zhao X, Luo Y, Kang P. Comparison of intravenous and topical dexamethasone for total knee arthroplasty: a randomized doubleblinded controlled study of effects on dexamethasone administration route and enhanced recovery. J Arthroplast. 2020:1237-43.

22. Srikandarajah S, Gilron I. Systematic review of movement-evoked pain versus pain at rest in postsurgical clinical trials and meta-analyses: a fundamental distinction requiring standardized measurement. Pain. 2011; 152(8):1734-9.

23. Marsh J, Bryant D, MacDonald SJ, Naudie D, Remtulla A, McCalden R, et al. Are patients satisfied with a web-based followup after total joint arthroplasty? Clin Orthop Relat Res. 2014;472(6):1972-81.

24. Woolf CJ. Central sensitization: implications for the diagnosis and treatment of pain. Pain. 2011;152(3 Suppl):S2-15.

25. Malfait AM, Schnitzer TJ. Towards a mechanism-based approach to pain management in osteoarthritis. Nat Rev Rheumatol. 2013;9(11):654-64.

26. DeLeo JA. Basic science of pain. J Bone Joint Surg Am. 2006;88(Suppl 2):5862

27. Lunn TH, Frokjaer VG, Hansen TB, Kristensen PW, Lind T, Kehlet H. Analgesic effect of perioperative escitalopram in high pain catastrophizing patients after total knee arthroplasty: a randomized, double-blind, placebo-controlled trial. Anesthesiology. 2015;122(4):884-94.

28. Lunn MP, Hughes RA, Wiffen PJ. Duloxetine for treating painful neuropathy, chronic pain or fibromyalgia. Cochrane Database Syst Rev. 2014:1:Cd007115.

29. Knadler MP, Lobo E, Chappell J, Bergstrom R. Duloxetine: clinical pharmacokinetics and drug interactions. Clin Pharmacokinet. 2011;50(5): 281-94

30. Leopold SS, Porcher R. Editorial: the minimum clinically important difference-the least we can do. Clin Orthop Relat Res. 2017:475(4):929-32.

31. Vendittoli PA, Makinen $P$, Drolet $P$, Lavigne $M$, Fallaha M, Guertin MC, et al. A multimodal analgesia protocol for total knee arthroplasty. A randomized, controlled study. J Bone Joint Surg Am. 2006;88(2):282-9.

32. Memtsoudis SG, Poeran J, Kehlet H. Enhanced recovery after surgery in the United States: from evidence-based practice to uncertain science? Jama. 2019;321(11):1049-50.

33. Vendittoli PA, Pellei K, Desmeules F, Massé V, Loubert C, Lavigne M, et al. Enhanced recovery short-stay hip and knee joint replacement program improves patients outcomes while reducing hospital costs. Orthopaedics Traumatol Surg Res. 2019;105(7):1237-43.

\section{Publisher's Note}

Springer Nature remains neutral with regard to jurisdictional claims in published maps and institutional affiliations. 